\title{
Correlation coefficients of generalized intuitionistic fuzzy sets by centroid method
}

\author{
I.M. Hanafy ${ }^{1}$, A. A. Salama ${ }^{2}$, K. M. A. Mahfouz ${ }^{3}$ \\ ${ }^{1,2,3}$ Department Of Mathematics and Computer Science, Faculty Of Science, Port Said University, Egypt
}

\begin{abstract}
In this paper, we propose a method to calculate the correlation coefficient of generalized intuitionistic fuzzy sets by means of "centroid". The value which obtained from this method tell us the strength of relationship between the generalized intuitionistic fuzzy sets [11] and the whether the generalized intuitionistic fuzzy sets are positively or negatively related. This method looks better than previous methods which only evaluated the strength of relation. Furthermore, we extended the "centroid" method to interval-valued generalized intuitionistic fuzzy sets. The value of the correlation coefficient between interval-valued generalized intuitionistic fuzzy sets lies in the interval [-1, 1], as computed from our formula.
\end{abstract}

Keywords: Centroid, Correlation coefficient, Interval-valued generalized intuitionistic fuzzy sets, Generalized intuitionistic fuzzy sets.

\section{Introduction}

In 1965 [[7]], Zadeh first introduced the concept of fuzzy sets. In many real applications to handle uncertainty, fuzzy set is very much useful and in this one real value $\mu_{A}(x) \in[0,1]$ is used to represent the grade of membership of a fuzzy set A defined on the universe of discorse $X$. After two decades Turksen and Yu.[[9],12] proposed the concept of interval-valued fuzzy set [8]. But for some applications [1,2,3,4] it is not enough to satisfy to consider only the membership-function supported by the evident but also have to consider the non-membership function against by the evident. Atanassov [[6]] introduced another type of fuzzy sets that is called intuitionistic fuzzy set (IFS) which is more practical in real life situations. Intuitionistic fuzzy sets handle incomplete information i.e., the grade of membership function and non-membership function but not the indeterminate information and inconsistent information which exists obviously in belief system. Salama [10] introduced and studied the neutrosophic sets and its operations as a generalized the intuitionistic fuzzy set.

Definition 1.1

Let $X$ be a fixed set. A fuzzy set $A$ of $X$ is an object having the form $A=\left\{\left(x, \mu_{A}(x)\right), x \in X\right\}$ where the function $\mu_{A}: X \rightarrow[0,1]$ define the degree of membership of the element $x \in X$ to the set $A$, which is a subset of $X$.

Definition 1.2

Let $X$ be a fixed set. An intuitionistic fuzzy set $A$ of $X$ is an object having the form $A=\left\{\left(x, \mu_{A}(x), v_{A}(x)\right), x \in X\right\}$ where the function: $\mu_{A}: X \rightarrow[0,1]$ and $v_{A}: X \rightarrow[0,1]$ define respectively the degree of membership and degree of non-membership of the element $x \in X$ to the set $A$, which is a subset of $X$ and for every $x \in X, 0 \leq \mu_{A}(x)+v_{A}(x) \leq 1$.

An element $x$ of $X$ is called significant with respect to a fuzzy subset $A$ of $X$ if the degree of membership $\mu_{A}(x)>0.5$, otherwise, it is insignificant. We see that for a fuzzy subset $A$ both the degrees of membership $\mu_{A}(x)$ and non-membership $v_{A}(x)=1-\mu_{A}(x)$ can not be significant. Further, for an intuitionistic fuzzy set $A=\left\{\left(x, \mu_{A}(x), v_{A}(x)\right), x \in X\right\}$ it is observe that $0 \leq \mu_{A}(x)+v_{A}(x) \leq 1$, for all $x \in X$ and hence it is observed that $\min \left\{x, \mu_{A}(x), v_{A}(x)\right\} \leq 0.5$, for all $x \in X$.

In statistics a concept called correlation is often used. Correlation analysis can be employed to study the nature of the relation between the variables.

\section{Correlation coefficient of fuzzy sets}

Suppose there is a fuzzy set $A \subseteq F$, where $F$ is a fuzzy space. The fuzzy sets $A$ defined on a crisp set $X$ with a membership function $\mu_{A}$, the probability of fuzzy set has been defined by Zadeh [[8]]

$$
P(A)=\int_{X} \mu_{A}(x) d P=E\left(\mu_{A}\right),
$$


where $P$ is the probability measure over $X$, and $E\left(\mu_{A}\right)$ is the mean of the membership function of a fuzzy event $A$.

When we have a random sample $\left(x_{1}, x_{2}, \ldots, x_{n}\right)$ from a crisp set $X$, with the membership function $\mu_{A}$ of some specific fuzzy set $A$, then the sample mean and sample variance of the membership function of $A$, defined on $X$, can be written as, (see[[1]]),

$$
\bar{\mu}_{A}=\frac{\sum_{i=1}^{n} \mu_{A}\left(x_{i}\right)}{n}, \quad S_{A}^{2}=\frac{\sum_{i=1}^{n}\left(\mu_{A}\left(x_{i}\right)-\bar{\mu}_{A}\right)^{2}}{n-1},
$$

where $\bar{\mu}_{A}$ and $S_{A}^{2}$ are the average and the degree of variations of membership function of fuzzy set $A$, then the correlation coefficient, $r_{A, B}$, between the fuzzy sets $A$ and $B$ :

$$
r_{A, B}=\frac{\sum_{i=1}^{n}\left(\mu_{A}\left(x_{i}\right)-\bar{\mu}_{A}\right)\left(\mu_{B}\left(x_{i}\right)-\bar{\mu}_{B}\right) / n-1}{S_{A} S_{B}} .
$$

\section{Correlation coefficient of intuitionistic fuzzy sets}

Yu [[12]] defined the correlation of $A$ and $B$ in the collection $F([a, b])$ of all fuzzy numbers whose supports are included in a closed interval $[a, b]$ as follows:

$$
C_{Y}(A, B)=\frac{1}{b-a} \int_{a}^{b} \mu_{A}(x) \mu_{B}(x)+v_{A}(x) v_{B}(x) d x,
$$

$0 \leq \mu_{A}(x)+v_{A}(x)=1$ and the correlation coefficient of fuzzy numbers $A, B$ was defined by

$$
\rho_{Y}=\frac{C_{Y}(A, B)}{\sqrt{C_{Y}(A, A) \times C_{Y}(B, B)}} .
$$

In 1991, Gerstenkorn and Manko [[2]] defined the correlation of intuitionistic fuzzy sets $A$ and $B$ in a finite set $X=\left\{x_{1}, x_{2}, \ldots, x_{n}\right\}$ as follows:

$$
C_{G M}(A, B)=\sum_{i=1}^{n}\left(\mu_{A}\left(x_{i}\right) \mu_{B}\left(x_{i}\right)+v_{A}\left(x_{i}\right) v_{B}\left(x_{i}\right)\right)
$$

correlation coefficient of fuzzy numbers $A, B$ was given by:

$$
\begin{aligned}
\rho_{G M} & =\frac{C_{G M}(A, B)}{\sqrt{T(A) \cdot T(B)}} \\
T(A) & =\sum_{i=1}^{n}\left(\mu^{2}{ }_{A}\left(x_{i}\right)+v^{2}{ }_{A}\left(x_{i}\right)\right) .
\end{aligned}
$$

In 1995, Hong and Hwang [[3]] defined the correlation of intuitionistic fuzzy sets $A$ and $B$ in a probability space $(X, B, P)$ as follows:

$$
C_{H H}(A, B)=\int_{X}\left(\mu_{A} \mu_{B}+v_{A} v_{B}\right) d P
$$

correlation coefficient of intuitionistic fuzzy numbers $A, B$ was given by

$$
\rho_{H H}=\frac{C_{H H}(A, B)}{\sqrt{C_{H H}(A, A) \cdot C_{H H}(B, B)}} .
$$

Hung and $\mathrm{Wu}[[4][5]]$ introduce the concept of positively and negatively correlated and used the concept of centroid to define the correlation coefficient of intuitionistic fuzzy sets which lies in the interval [$1,1]$, and the correlation coefficient of intuitionistic fuzzy sets $A$ and $B$ was given by: 


$$
\begin{array}{ll}
\rho_{H W}=\frac{C_{H W}(A, B)}{\sqrt{C_{H W}(A, A) \cdot C_{H W}(B, B)}}, & \\
C_{H W}=m\left(\mu_{A}\right) m\left(\mu_{B}\right)+m\left(v_{A}\right) m\left(v_{B}\right) & \\
m\left(\mu_{A}\right)=\frac{\int x \mu_{A}(x) d x}{\int \mu_{A}(x) d x} & m\left(v_{A}\right)=\frac{\int x v_{A}(x) d x}{\int v_{A}(x) d x} \\
m\left(\mu_{B}\right)=\frac{\int x \mu_{B}(x) d x}{\int \mu_{B}(x) d x} & m\left(v_{A}\right)=\frac{\int x v_{B}(x) d x}{\int v_{B}(x) d x}
\end{array}
$$

\section{Correlation coefficient of generalized intuitionistic fuzzy sets}

The correlation coefficient between generalized intuitionistic fuzzy sets ranges in [-1,1], which can correlate fuzzy concepts. Thus, we propose the following correlation measure.

\section{Definition $4.1[[11]]$}

Let $X$ be a fixed set. A generalized intuitionistic fuzzy set (GIFS) $A$ of $X$ is an object having the form $A=\left\{\left(x, \mu_{A}(x), v_{A}(x)\right), x \in X\right\}$ where the function $\mu_{A}: X \rightarrow[0,1]$ and $v_{A}: X \rightarrow[0,1]$ define respectively the degree of membership and degree of non-membership of the element $x \in X$ to the set $A$, which is a subset of $X$ and for every $x \in X$ satisfy the condition $\mu_{A}(x), v_{A}(x) \leq 0.5$ for all $x \in X$. This condition is called generalized intuitionistic condition (GIC). In fact, all generalized intuitionistic fuzzy sets are intuitionistic fuzzy set but all intuitionistic fuzzy sets are not generalized intuitionistic fuzzy sets.

\section{Definition 4.2}

Let $A, B$ are generalized intuitionistic fuzzy sets, we define

$$
C(A, B)=m\left(\mu_{A}\right) m\left(\mu_{B}\right)+m\left(v_{A}\right) m\left(v_{B}\right)
$$

(4.1) and we

call it correlation formula of $A$ and $B$. furthermore, we call

$$
\begin{aligned}
& \rho(A, B)=\frac{C(A, B)}{\sqrt{C(A, A) \cdot C(B, B)}}, \\
& m\left(\mu_{A}\right)=\frac{\int x \mu_{A}(x) d x}{\int \mu_{A}(x) d x} \\
& m\left(v_{A}\right)=\frac{\int x v_{A}(x) d x}{\int v_{A}(x) d x} \\
& m\left(\mu_{B}\right)=\frac{\int x \mu_{B}(x) d x}{\int \mu_{B}(x) d x} \\
& m\left(v_{A}\right)=\frac{\int x v_{B}(x) d x}{\int v_{B}(x) d x}
\end{aligned}
$$

where

are the centroid of $\mu_{A}, v_{A}, \mu_{B}$ and $v_{B}$, respectively.

\section{Proposition 4.1}

For all $A$ and $B$ are generalized intuitionistic fuzzy sets, we have

i. $C(A, B)=C(B, A)$.

ii. $\rho(A, B)=\rho(B, A)$.

iii. If $A=B$, then $\rho(A, B)=1$. 
Theorem 4.1

For all $A$ and $B$ are generalized intuitionistic fuzzy sets, we have

$$
|\rho(A, B) \leq 1| .
$$

Proof

By the Cauchy-Schwarz inquality, Therefore, we have $\rho(A, B) \leq 1$.

\section{Corollary 4.1}

Let $A$ and $B$ are generalized intuitionistic fuzzy sets satisfied with $\mu_{A}=c \mu_{B}$ and $v_{A}=c v_{B}$ for an arbitrary real number $c>0$, then $\rho(A, B)=1$.

\section{Conclusion}

Our main goal of this work is propose a method to calculate the correlation coefficient of generalized intuitionistic fuzzy sets by means of "centroid" which lies in [-1,1], give us information for the degree of the relationship between the generalized intuitionistic fuzzy sets and the fact that these two sets are positively or negatively related.

\section{References}

[1] Ding-An Chiang and Nancy P. Lin, (1997), "Correlation of fuzzy sets", Fuzzy Sets and Systems, 102: 221-226.

[2] Gerstenkorn, T., Manko, J., (1991), "Correlation of intuitionistic fuzzy sets". Fuzzy Sets and Systems, 44, 39-43.

[3] Hong, D. H. and Hwang, S. W., (1995), "Correlation of intuitionistic fuzzy sets in probability spaces". Fuzzy Sets and Systems, 75: 77-81.

[4] Hung, W. L., (2001), "Using statistical viewpoint in developing correlation of intuitionistic fuzzy sets". International Journal of Uncertainty Fuzziness Knowledge Based Systems, 9: 509-516.

[5] Hung, W. L. and Wu, J. W., (2002), "Correlation of intuitionistic fuzzy sets by centroid method". Information Sciences, 144: 219-225.

[6] K. Atanassov, (1986), "Intuitionistic fuzzy sets". Fuzzy Sets and Systems, 20:87-96.

[7] L. A. Zadeh, (1965), "Fuzzy sets". Information and Control, 8: 338-353.

[8] L. A. Zadeh, (1968), "Probability measures of fuzzy events", Journal of Mathematical Analysis and Applications, $23: 421-427$.

[9] Turksen, I., (1986), "Interval-valued fuzzy sets based on normal form". Fuzzy Sets and Systems, 20: $191-210$.

[10] A.A.Salam and S.A.Alblowi "NEUTROSOPHIC SET AND NEUTROSOPHIC TOPOLOGICAL SPACES" Accepted in International Organization of Scientific Research (IOSR) M. Journal 2012.

[11] T. K. Mondal and S. K. Samanta, (2002), "Generalized intuitionistic fuzzy sets". The Journal of Fuzzy Mathematics, 10(4): 839862 .

[12] Yu, C., (1993), "Correlation of fuzzy numbers". Fuzzy Sets and Systems, 55: 303-307. 